\title{
High rate of IIA/IIB neck groups involvement supports complete lateral neck dissection in thyroid carcinoma
}

\author{
Emilien Chebib ${ }^{1}$, Caroline Eymerit ${ }^{2}$, Nathalie Chabbert-Buffet ${ }^{3}$, Bruno Angelard ${ }^{1}$, Jean Lacau St Guily ${ }^{1,4}$, \\ Sophie Périé ${ }^{1,5}$
}

${ }^{1}$ Department of Otolaryngology Head and Neck Surgery, Tenon Hospital, Assistance Publique Hôpitaux de Paris (APHP), and Faculty Medicine Sorbonne University, Paris, France; ${ }^{2}$ Department of Pathology, Tenon Hospital, Assistance Publique Hôpitaux de Paris (APHP), and Faculty Medicine Sorbonne University, Paris, France; ${ }^{3}$ Department of Endocrinology and Reproductive Medicine, Pregnancy Associated Cancer Network, Tenon Hospital, Assistance Publique Hôpitaux de Paris (APHP), and Faculty Medicine Sorbonne University, Paris, France; ${ }^{4}$ Department of Otolaryngology Head and Neck Surgery, Rothschild Foundation, Paris, France; ${ }^{5}$ Department of Otolaryngology Head and Neck Surgery, Com Maillot - Hartmann Clinic, Neuilly Sur Seine, France

Contributions: (I) Conception and design: S Périé, N Chabbert-Buffet, J Lacau St Guily; (II) Administrative support: All authors; (III) Provision of study materials or patients: All authors; (IV) Collection and assembly of data: E Chebib, S Périé, C Eymerit; (V) Data analysis and interpretation: E Chebib, S Périé, C Eymerit; (VI) Manuscript writing: All authors; (VII) Final approval of the manuscript: All authors.

Correspondence to: Sophie Périé, MD. Department of Otolaryngology Head and Neck Surgery - Hartmann Clinic, Com Maillot - 26 Boulevard Victor Hugo; 92200 Neuilly Sur Seine, France. Email: prsophieperie@gmail.com.

Background Although therapeutic lateral neck dissection (LND) may be indicated in thyroid carcinoma, the cervical lymph node groups IIA and IIB, according to Robbins classification, are often not removed. The aim of our study was to determine the relevance of complete comprehensive LND in thyroid carcinoma.

Methods: We conducted a retrospective study between January 2011 and August 2018 in a university teaching hospital. Histopathological analysis of LND performed during total thyroidectomy in thyroid carcinoma was reviewed according to lymph node level. To demonstrate that neck dissection of upper groups, IIA/IIB, is necessary when therapeutic LND is indicated in thyroid carcinoma, we compared histopathological involvement in complete comprehensive LND of the upper groups IIA/IIB to the lower groups III/IV/V.

Results: A total of 30 LND (24 patients) were suitable for analysis. Analysis by neck side revealed that comprehensive LND dissection samples were negative in 3 cases, and positive in 27 . In those 27 positive LND, 15 demonstrated involvement of the groups IIA/IIB, and 12 were positive for involvement of the III/ IV/V groups only. The combined presence of positive IIA/IIB and positive III/IV/V was observed in 15 of the 27 neck sides. There was no positive IIA/IIB without positive involvement of III/IV/V groups.

Conclusions: The high rate of positive cervical lymph nodes in the upper groups IIA/IIB supports complete comprehensive LND rather than selective III/IV/V LND in thyroid carcinoma surgery. However, impact on survival and whether postoperative radioactive iodine treatment may be modulated remain to be studied.

Keywords: Lateral neck dissection (LND); pathological analysis; thyroid cancer; thyroid carcinoma neck metastasis

Submitted Apr 10, 2020. Accepted for publication Sep 30, 2020.

doi: $10.21037 /$ gs-20-443

View this article at: http://dx.doi.org/10.21037/gs-20-443 


\section{Introduction}

\section{Background}

Prophylactic (N0 neck) or therapeutic neck dissection (N+ neck) in thyroid carcinoma is a current topic of discussion. It has been shown that central neck dissection (central ND), according to Robbins' classifications $(1,2)$, should be routinely combined with thyroidectomy in medullary thyroid carcinoma (MTC), in papillary carcinoma and microcarcinoma with aggressive criteria in order to decrease the risk of recurrence (3-6), to allow accurate disease staging, and to determine whether postoperative radioactive iodine treatment is needed in differentiated thyroid carcinoma (DTC) $(3,5,7)$.

Prophylactic lateral neck dissection (LND) is well established for MTC, depending on the preoperative thyrocalcitonin rate (6). In DTC, the management and the prognostic role of lymph nodes remains controversial, but the gold standard is to perform therapeutic LND and not prophylactic (8). This limits cosmetic sequelae and functional complications (9-11) despite the lack of oncologic benefit in N0 patients (12-14). Indication of therapeutic LND in DTC is commonly discussed on thyroid cancer staging and patient age, when there is preoperative ultrasound evidence of lateral lymph node metastasis, and by results of fine needle aspiration (FNA) in cases of "borderline" or "suspicious" lymph nodes (3,5-7,12,15-17). In some cases of DTC, a two-stage surgical management can be preferred based on histopathological criteria from initial thyroidectomy and central ND $(3,11)$. Others surgeons perform LND in cases of positive intraoperative frozen sections on suspicious lateral lymph nodes (18).

It was previously reported that cervical lymph node metastasis did not have a major impact on survival, and some consider that the extent of neck dissection did not predict regional recurrence in DTC patients with lateral neck metastases (19). However, recent studies demonstrated that patients managed by therapeutic comprehensive LND have a lower rate of recurrence than patients managed by therapeutic selective LND (20-22).

\section{Particular features of neck dissection in thyroid carcinoma}

In thyroid carcinoma, extents of therapeutic LND are described as comprehensive or selective (19-21,23). Levels IIA to V (IIA, IIB, III, IV, V groups according to Robbins' classification) are removed during comprehensive LND, but selective LND commonly removes levels III, IV and
VB, but not the IIA/IIB groups $(1,2,8,9,15,20,22,23)$. Comprehensive LND may be indicated in thyroid carcinoma, as metastatic thyroid carcinoma disease may involve all of these levels (9,21-23). This also allows removal of group V during complete dissection of levels III and IV to the posterior cervical roots. In addition, most studies do not distinguish levels VA and VB as it is difficult to study them separately (15). It is also important to point out that group IIB (retrospinal) was initially termed as group VA in the Robbins classification $(1,8)$ and isolated behind the sternocleidomastoid muscle $(1,2,8,9)$.

\section{Study objectives in therapeutic LND}

The extent of therapeutic LND, selective or comprehensive, has been subject to debate over the past 40 years. In several series with LND performed, including also MTC, selective resection of only lower groups III/IV and V $(16,24)$, were preferred to limit the surgical morbidity. The aim of our work was to study the relevance of dissection of all lateral groups by performing a comprehensive LND, when therapeutic LND is indicated in thyroid carcinoma, removing levels IIA/IIB, III, IV, and V. The originality was to make comparison between lower III/IV/V groups to upper IIA/IIB involvement. Analysis of histopathological results of LND according to upper IIA/IIB or lower III/IV/ $\mathrm{V}$ groups, was conducted in order to support subsequent complete comprehensive LND instead of selective LND (lower groups) in thyroid carcinoma.

We present the following article in accordance with the STROBE reporting checklist (available at http://dx.doi. org/10.21037/gs-20-443).

\section{Methods}

\section{Study design, setting and etbics}

This was a retrospective study of data from patients followed up at the Department of Otolaryngology Head and Neck Surgery in Tenon hospital (Paris, France) from January 2009 to August 2018. The study was conformed to the ethical principles for medical research involving human subjects of the Declaration of Helsinki (as revised in 2013). This retrospective study and the individual consent waived were approved by the Commission d'Evaluation et de Recherche Observationnelle en OtoRbinoLaryngologie (CEORL: Ethics Committee of the Society of Otolaryngology, France; number 2020-04-001- 
SP) in accordance with French legislation (Public Health Code amended by law No 2004-806, August 9, 2004. Data were strictly anonymous, collected during the end of 2018 , from papers or files containing detailed medical information of patients.

\section{Study population, selection criteria}

All patients treated consecutively for thyroid carcinoma with comprehensive LND during the study period were included.

Criteria for inclusion were patients surgically treated for thyroid cancer by a single experienced thyroid surgeon, by total or completion thyroidectomy with central ND, associated with a comprehensive LND (removing the IIA/IIB/III/IV/V groups) on at least one side. This comprehensive complete LND was required for preoperative identified $\mathrm{N}+$ on lateral compartment in DTC, and in all MTC (high thyrocalcitonin rate). Patients previously operated by total thyroidectomy and central ND, who required LND in a second step, were also included (tumors presented aggressive criteria and/or severe $\mathrm{pN}+$ involved in central ND).

Criteria of exclusion were patients with no preoperative lateral $\mathrm{N}$, patients who required only central ND with total thyroidectomy, and also patients with completion of LND initially performed in another center.

All patients underwent preoperative thyroid and neck ultrasonography. Diagnosis of DTC or MTC was suspected preoperatively by thyroid FNA cytology and thyrocalcitonin level, and intraoperatively, on frozen section, or at definitive pathological analysis. Surgery was performed in one or several steps, as the diagnosis of cancer can only be confirmed on definitive histopathological examination, or in patients who had previously undergone incomplete thyroidectomy or neck dissection. The decision to perform comprehensive LND or not, was based on the results of the preoperative thyroid staging, physical neck presentation, neck ultrasonography (identified $\mathrm{N}+$ on the lateral compartment), including FNA of "borderline" or "suspicious" lymph nodes in the most recent period.

Total thyroidectomy or completion thyroidectomy was performed prior to central ND and LND. Central ND was performed from the body of the hyoid bone superiorly, the carotid sheath laterally and suprasternal notch inferiorly (level VI/VII). LND consisted of a comprehensive LND, removing levels IIA, IIB, III, IV, and V $(2,8)$.

\section{Study variables}

Thyroidectomy specimens and neck dissections were carefully oriented by the surgeon, according to the Robbins standardized neck dissection terminology, marked on a diagram and sent for histopathological examination. Specimens were fixed in formaldehyde. Each cervical lymph node was entirely sectioned every $3 \mathrm{~mm}$ and $5 \mu \mathrm{m}$. Sections were mounted and stained by haematoxylin-eosinsaffron (HES) and then examined by light microscopy. Papillary carcinoma and cervical lymph node metastasis were diagnosed when typical nuclear atypia was observed. In cases of follicular carcinoma, thyroglobulin antibody immunohistochemistry was sometimes performed.

\section{Statistical analysis}

The mean number and mean size of cervical lymph nodes examined were recorded. Results were scored as pathological $\mathrm{pN}+$ for lymph node involvement or $\mathrm{pN}$ - for no involvement. The lymph node capsular status was also reported (extracapsular spread or not). These data allowed staging of patients according to pTN classification (7).

The pathological results and correlations between upper and lower levels of lymph nodes resected (IIA/IIB, versus III/IV/V) were described according to upper or lower level and noted LND+ (positive LND) or LND- (negative LND).

\section{Results}

\section{Participants: baseline characteristics and histopatbology of} thyroid carcinoma

Twenty-four consecutive patients (14 males, 10 females; mean age: 47.46 years; range: 22 to 75 years) were included in this retrospective study. Flow diagram and baseline characteristics of patients are reported in Figure 1 and Table 1. Patient population was mostly male (58\%) (Table 1). Among the 24 patients, there were 25 thyroid carcinomas; one patient presented both MTC (T1) and DTC (T1). There were 6 T1, 8 T2, 10 T3 and 1 T4 based on thyroid carcinoma staging (7). Mean MTC preoperative thyrocalcitonin level was $1,635 \mathrm{pg} / \mathrm{mL}$ (range, $157-$ $5,500 \mathrm{pg} / \mathrm{mL}$ ). Bilateral central ND was performed in all patients (48 central ND).

Out of the 30 LNDs, comprehensive LND was performed on 24 patients, ipsilateral LND on 18 patients, and bilateral on 6 patients (30 LNDs total). In 3 cases FNA 


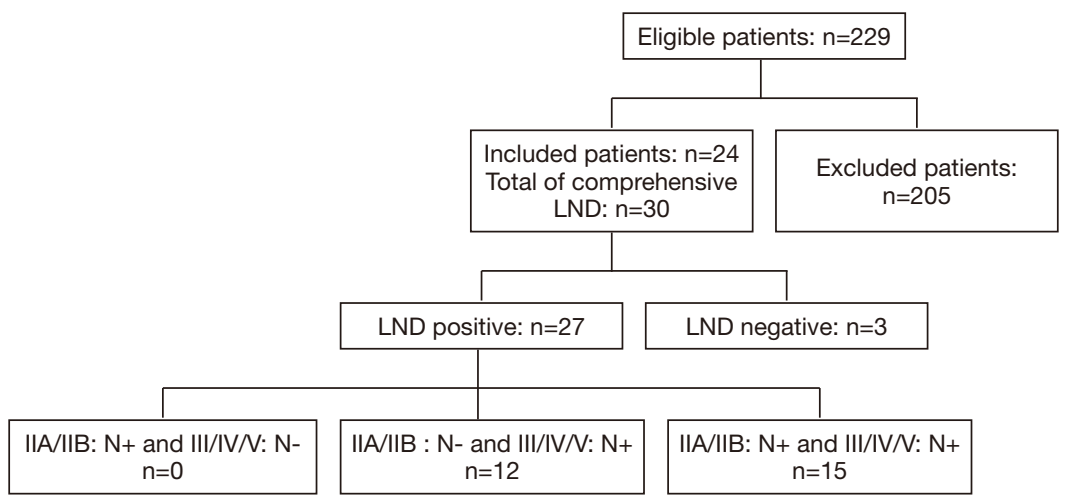

Figure 1 Flow diagram. +, positive; -, negative.

Table 1 Patient characteristics

\begin{tabular}{|c|c|}
\hline Population characteristics & $\mathrm{n}[\%]$ \\
\hline Mean age $(n=24)$ & 47.46 years \\
\hline \multicolumn{2}{|l|}{$\operatorname{Sex}(n=24)$} \\
\hline Male & $14[58]$ \\
\hline Female & $10[42]$ \\
\hline \multicolumn{2}{|l|}{ Type of tumor $(n=25)^{\dagger}$} \\
\hline Differentiated thyroid carcinoma (DTC) & $19[76]$ \\
\hline Medullary thyroid carcinoma (MTC) & $6[24]$ \\
\hline \multicolumn{2}{|c|}{ T staging (according to the AJCC 2017 TNM classification) ${ }^{\ddagger}$} \\
\hline $\mathrm{T} 1$ & $6[24]$ \\
\hline $\mathrm{T} 2$ & 8 [32] \\
\hline T3 & $10[40]$ \\
\hline $\mathrm{T} 4$ & $1[4]$ \\
\hline \multicolumn{2}{|l|}{ Intervention type on $\mathrm{T}(\mathrm{n}=24)$} \\
\hline $\begin{array}{l}\text { Total thyroidectomy, central ND and } \\
\text { LND in one step }\end{array}$ & $14[58]$ \\
\hline $\begin{array}{l}\text { Total thyroidectomy, central ND and } \\
\text { LND in two or more steps }{ }^{\S}\end{array}$ & 10 [42] \\
\hline \multicolumn{2}{|l|}{ Cervical neck dissection $(n=30)$} \\
\hline Bilateral CND & 24 [100] \\
\hline Unilateral LND & $18[75]$ \\
\hline Bilateral LND & $6[25]$ \\
\hline
\end{tabular}

${ }^{\dagger}$, one patient had simultaneously medullary thyroid carcinoma and differentiated thyroid carcinoma; ${ }^{\ddagger}$, regrouping DTC and MTC; ${ }^{\S}$, one patient had total thyroidectomy, central ND and LND in three steps. CND, central neck dissection; LND, lateral neck dissection. found "borderline" lymph nodes on one neck side.

A one step LND and thyroidectomy surgical approach was performed on 14 patients ( $\mathrm{N}$ identified on the central or lateral compartment at the preoperative thyroid and neck ultrasonography) and a two-steps surgery with LND (multiple $\mathrm{pN}+$ at the histopathological examination of the central ND), was performed for the remaining 10 .

\section{Histopathology of lymph node dissections (Tables 2,3)}

A total of 30 comprehensive LND from 24 patients were studied to compare involvement of upper groups IIA/IIB and lower groups III/IV/V (Table 2) of cervical lymph nodes. Three LND demonstrated no positive cervical lymph node (those 3 patients have "suspicious" lymph nodes at US, but no FNA was performed).

The number of lymph nodes included in the positive LND group (including three with positive lymph nodes obtained at FNA) ranged from 10 to 60 nodes (mean: 31.45 ) and the number of positive lymph nodes ranged from 1 to 15 (mean: 5.0). At least 65 lymph nodes presented signs of extracapsular spread in the 27 positive LND group (21 patients) (Table 3).

Staging according to the pTNM classification found 3 $\mathrm{N} 1 \mathrm{a}$ and $21 \mathrm{~N} 1 \mathrm{~b}$ nodes in the 24 patients.

\section{Involvement of the ILA/IIB and III/IV/V groups according to upper or lower level, at neck side}

Analysis according to upper or lower level showed that among the 27 LND positive lymph node groups practiced on 21 patients, $15(55.5 \%)$ LND were positive in the IIA 
Table 2 Histopathological correlation between upper and lower cervical nodes groups in the comprehensive lateral neck dissection (LND) at neck side in the 24 patients (19 cases of differentiated thyroid carcinoma and 6 cases of medullary thyroid carcinoma)

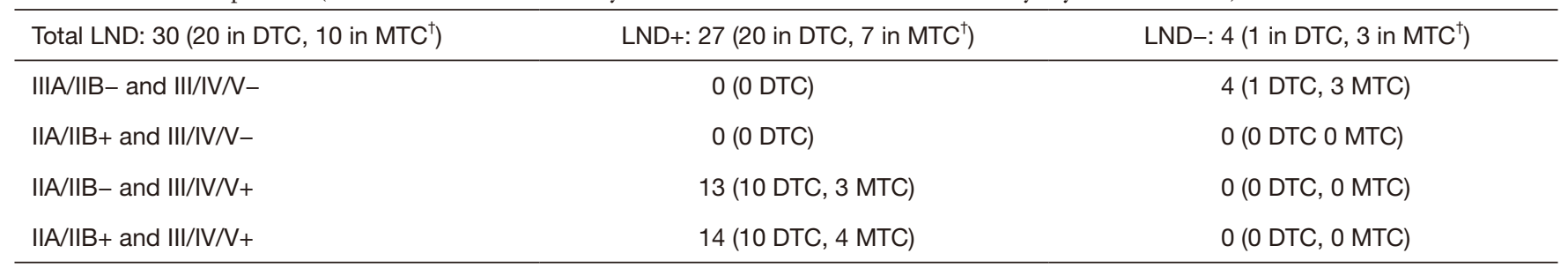

${ }^{\dagger}$, one patient had simultaneously medullary thyroid carcinoma and differentiated thyroid carcinoma. He had one LND which was positive for DTC (IIA and IV) and negative for MTC. +, positive; -, negative. DTC, differentiated thyroid carcinoma; MTC, medullary thyroid carcinoma.

Table 3 Histopathological characteristics of $\mathrm{pN}+$ in the comprehensive lateral neck dissection (LND) in differentiated thyroid carcinoma and medullary thyroid carcinoma

\begin{tabular}{lccc}
\hline $\begin{array}{l}\text { LND+ } \\
(\mathrm{n}=27)\end{array}$ & $\begin{array}{c}\text { Number+ } \\
(\text { mean })\end{array}$ & $\begin{array}{c}\text { Size+ (mean, } \\
\mathrm{mm})\end{array}$ & $\begin{array}{c}\text { Extracapsular spread in } \\
\text { different groups }(\%)\end{array}$ \\
\hline IIA & 2.72 & 12.3 & 50 \\
IIB & 2.2 & 9 & 73 \\
III/VA & 2.63 & 11.2 & 40 \\
IV/VB & 1.81 & 11.3 & 60 \\
\hline
\end{tabular}

+ , positive. DTC, differentiated thyroid carcinoma; MTC, medullary thyroid carcinoma.

and/or IIB groups and in III/IV/V groups, and 12 (45\%) were only positive in III/IV/V groups. No positive LND was positive in the IIA/IIB groups but negative in the III/ IV/V groups (Table 2).

\section{Histopathological characteristics of positive lymph nodes}

Histopathological characteristics of positive lymph nodes allowed us to show that when the upper IIA and/or IIB groups were positive, the average number and size of positive lymph nodes was high in the upper group IIA/IIB. We also found a $73 \%$ rate of extracapsular spread among the positive lymph nodes in the IIA/IIB groups, which was more than in the III/IV/V groups (Table 3).

\section{Analysis of positive LND in MTC (6 patients) and DTC (19 patients)}

Histopathological results of comprehensive LND and characteristics of positive lymph nodes in DTC and MTC were studied. Note that the patient who had both MTC and
DTC had only DTC metastasis in the LND performed.

The mean number and size of positive groups in DTC was 2.8 nodes of $12.25 \mathrm{~mm}$ (IIA), 1 node of $11 \mathrm{~mm}$ (IIB), 2.6 nodes of $10.8 \mathrm{~mm}$ (III/VA), 1.7 nodes of $10.4 \mathrm{~mm}$ (IV/VB), respectively. The mean number and size of positive groups in MTC was 3 nodes of $17.4 \mathrm{~mm}$ (IIA), 3 nodes of $20.6 \mathrm{~mm}$ (IIB), 3.6 nodes of $16.3 \mathrm{~mm}$ (III/VA), 2.3 nodes of $13 \mathrm{~mm}$ (IV/VB), respectively.

The extracapsular spread (\%) of $\mathrm{pN}+$ in the LND+ DTC was between 0 and $52.9 \%$ [ $46.4 \%$ in IIA (Figure 2), $0 \%$ in IIB, $25.8 \%$ in III/VA, $52.9 \%$ in IV/VB, respectively].

The extracapsular spread (\%) of $\mathrm{pN}+$ in the LND+ in MTC was between $50 \%$ and $88.9 \%$ (50\% in IIA, $88.9 \%$ in IIB, $66.7 \%$ in III/VA, 71.4\% in IV/VB, respectively).

\section{Discussion}

The aim of our work was to study the relevance of dissection of all lateral groups, the upper IIA/IIB as well as the lower III/IV/V groups, when therapeutic LND is indicated in thyroid carcinoma. Histopathological results of comprehensive LND performed in the present study according to upper or lower levels, showed a high rate of IIA/IIB lymph node involvement compared to III/IV/ $\mathrm{V}$ levels. This advocates therapeutic LND by complete comprehensive, rather than not selective lower III/IV/V LND, in thyroid carcinoma.

In DTC, LND is actually not prophylactic but therapeutic (22), and is discussed in views of preoperative check up by ultrasonography and FNA in "borderline" or "suspicious" lymph nodes in the lateral neck (3,5-7,12,15-17). The three negative comprehensive LND in this study were performed in patients who had no FNA on "suspicious" lateral lymph nodes in ultrasonography at the beginning of the study; this supports the 


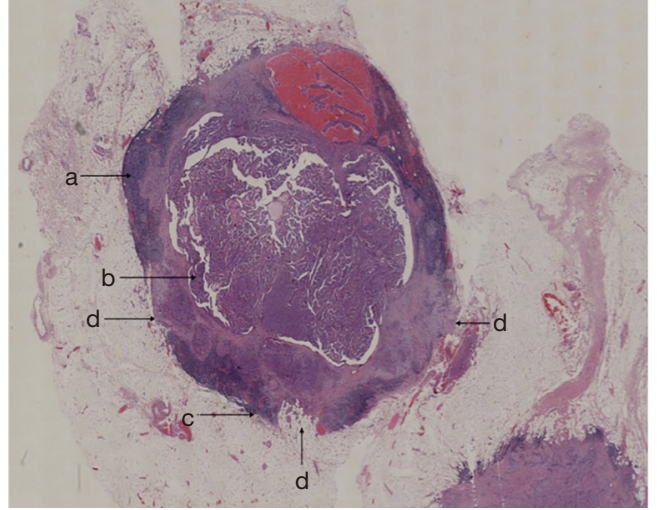

Figure 2 Lymph node metastasis of a papillary thyroid carcinoma with extracapsular spread. HES staining, digital slide scanned, magnification $\times 10$. (a) Normal lymph node tissue; (b) metastasis of papillary thyroid carcinoma; (c) lymph node's capsule; (d) extracapsular invasion of lymph node.

recommendation of the ATA, to avoid unnecessary LND in $\mathrm{N} 0$ patients $(5,6)$. For MTC, indication of complete comprehensive LND of the levels I to $\mathrm{V}$, is required when the preoperative thyrocalcitonin rate is significant, more than $200 \mathrm{pg} / \mathrm{mL}$ (6). Patients with MTC were accordingly included in the study since the aim was to analyse the levels of nodes involved in thyroid carcinoma, and like in DTC, some surgeons don't perform complete comprehensive LND in MTC, although LND is indicated (6).

LND commonly includes dissection of levels IIA, IIB, III and IV/V (comprehensive LND) in head and neck carcinoma (25). In thyroid carcinoma, some surgeons have preferred a selective LND, removing only the III/IV/V groups $(8,16,24)$, to limit morbidity of LND (large incisions with cosmetic consequences in young patients, lesions of accessory nerve with shoulder weakness, chronic neck pain, facial, phrenic, hypoglossal and sympathetic nerve damage, chyle leakage, salivary fistula and respiratory distress) (9-11). Authors also suggest that neck dissection should include level IIB whenever level IIA lymph node metastasis is found (26), but others claim dissection of levels II through $\mathrm{V}$ is required for optimal treatment in patients with lateral lymph nodes (8,21-23).

The aim of this study was not to discuss indications of LND in thyroid carcinoma $(3,5,7,12,16,17)$, but to study the relevance of a dissection including all levels of lateral cervical nodes when LND is indicated (i.e., comprehensive LND, including groups VA/VB since dissection of levels
III/IV was performed until the posterior cervical roots). The choice to further analyse the involved cervical nodes by upper or lower level was appropriate since we aimed to study the location of cervical metastasis in thyroid carcinoma, according to Robbins classification $(1,2)$. These results point out that when upper IIA/III groups were involved, so were the lower III/IV/V groups. There was no case of isolated IIA/IIB group involvement without positive III/IV/V group in our study. The group $\mathrm{V}$ was linked in this study with the lower groups, since the Robbins classification $(1,2,8)$ evolved and a lot of studies included them in the III and IV levels; in addition, the nodes around the levels III/IV are not always easy to distinguish from the VA/VB groups (15) and were addressed in this study with the levels III/IV for pathological analysis (III/VA level, and IV/VB level).

Extracapsular node spread is also frequent in all positive cervical nodes, ranging from $40 \%$ to $73 \%$. This high rate may be due to therapeutic and not prophylactic LND, which is currently performed for DTC $(3,5,7,12,16,17)$. In addition, the number of $\mathrm{pN}+$ in the IIA group was high and extracapsular spread was frequent in the IIA group in DTC. In contrast, all node groups were involved in MTC, with more than $50 \%$ of extracapsular spread in all groups $(50 \%$ to $88 \%$ ).

Our study shows that complete comprehensive and not selective LND in thyroid carcinoma is relevant not only for MTC but also for DTC. This agrees with previous studies on thyroid carcinoma (18) that point out complete comprehensive LND with central ND (3,5,7,8,12,16-18) is required for DTC. When indication of therapeutic LND is established (either preoperative lateral lymph node, or as a second step in aggressive DTC, or in MTC), dissection of only groups III/IV/V $(8,16,24)$ is insufficient, considering the high rate of lymphatic metastasis in groups II. In addition, unilateral or bilateral comprehensive LND was performed secondarily in some cases, if necessary, after obtaining pathology results of the initial surgery since massive involvement of central lymph nodes increases the rate of positive LND $(3,10,11)$ (8 and 5 patients in our study); metastases commonly start by involving the central nodes and subsequently spread to lateral nodes (except in cases located at the superior part of the thyroid) (27). LND can also help in identifying risk factors for recurrence in thyroid cancer (28).

Furthermore, when recurrent disease is located in undissected cervical nodes during a first selective LND, the secondary completion of the LND is more difficult due to 
fibrosis around level III previously dissected, next to the IIA/IIB groups.

The limits of the present study where a poor number of patients with a lack of statistical evidence, and the type of study which is a retrospective analysis. Nevertheless, our study is original in comparing the upper IIA/IIB and the lower III/IV/V cervical lymph nodes groups involved in thyroid carcinoma. Predicting factors of level IIA/IIB metastasis, impact of complete comprehensive LND on survival rate and on modulation of postoperative radioactive iodine treatment remains to be studied in a larger prospective analysis, keeping into account that cosmetic and functional sequels remain a real challenge in view of oncologic benefits.

\section{Conclusions}

A high prevalence of positive involvement of IIA/IIB groups during therapeutic LND for thyroid carcinoma may support to perform complete comprehensive LND including the upper IIA/IIB groups, instead of selective lower III/IV/V groups. These results should be studied in a larger population to confirm results and to evaluate impact on survival for patients.

\section{Acknowledgments}

The authors are grateful to Maria-Pia Tuset (MD, PhD) for his helpful linguistic contribution during the reading of the manuscript.

Funding: None.

\section{Footnote}

Reporting Checklist: The authors have completed the STROBE reporting checklist. Available at http://dx.doi. org/10.21037/gs-20-443

Data Sharing Statement: Available at http://dx.doi. org/10.21037/gs-20-443

Peer Review File: Available at http://dx.doi.org/10.21037/gs20-443

Conflicts of Interest: All authors have completed the ICMJE uniform disclosure form (available at http://dx.doi. org/10.21037/gs-20-443). The authors have no conflicts of interest to declare.

Ethical Statement: The authors are accountable for all aspects of the work in ensuring that questions related to the accuracy or integrity of any part of the work are appropriately investigated and resolved. The study was conformed to the ethical principles for medical research involving human subjects of the Declaration of Helsinki (as revised in 2013). This retrospective study and the individual consent waived were approved by the Commission d'Evaluation et de Recherche Observationnelle en OtoRbinoLaryngologie (CEORL: Ethics Committee of the Society of Otolaryngology, France; number 2020-04-001$\mathrm{SP}$ ) in accordance with French legislation (Public Health Code amended by law No 2004-806, August 9, 2004. Data were strictly anonymous, collected during the end of 2018, from papers or files containing detailed medical information of patients.

Open Access Statement: This is an Open Access article distributed in accordance with the Creative Commons Attribution-NonCommercial-NoDerivs 4.0 International License (CC BY-NC-ND 4.0), which permits the noncommercial replication and distribution of the article with the strict proviso that no changes or edits are made and the original work is properly cited (including links to both the formal publication through the relevant DOI and the license). See: https://creativecommons.org/licenses/by-nc-nd/4.0/.

\section{References}

1. Robbins KT, Clayman G, Levine PA, et al. Neck dissection classification update: revisions proposed by the American Head and Neck Society and the American Academy of Otolaryngology Head and Neck Surgery. Arch Otolaryngol Head Neck Surg 2002;128:751-8.

2. Robbins KT, Shaha AR, Medina JE, et al. Consensus statement on the classification and terminology of neck dissection. Arch Otolaryngol Head Neck Surg 2008;134:536-8.

3. Guerrier B, Berthet JP, Cartier C, et al. French ENT Society (SFORL) practice guidelines for lymphnode management in adult differentiated thyroid carcinoma. Eur Ann Otorhinolaryngol Head Neck Dis 2012;129:197-206.

4. Gonçalves Filho J, Zafereo ME, et al. Decision making for the central compartment in differentiated thyroid cancer. 
Eur J Surg Oncol 2018;44:1671-8.

5. Haugen BR, Alexander EK, Bible KC, et al. 2015 American Thyroid Association Management Guidelines for Adult Patients with Thyroid Nodules and Differentiated Thyroid Cancer: The American Thyroid Association Guidelines Task Force on Thyroid Nodules and Differentiated Thyroid Cancer. Thyroid 2016;26:1-133.

6. Wells SA, Asa SL, Dralle H, et al. Revised American Thyroid Association guidelines for the management of medullary thyroid carcinoma. Thyroid 2015;25:567-610.

7. Lamartina L, Grani G, Arvat E, et al. 8th edition of the AJCC/TNM staging system of thyroid cancer: What to expect (ITCO\#2). Endocr Relat Cancer 2018;25:L7-L11.

8. Javid M, Graham E, Malinowski J, et al. Dissection of Levels II Through V Is Required for Optimal Outcomes in Patients with Lateral Neck Lymph Node Metastasis from Papillary Thyroid Carcinoma. J Am Coll Surg 2016;222:1066-73.

9. Roh JL, Kim JM, Park CI. Lateral cervical lymph node metastases from papillary thyroid carcinoma: Pattern of nodal metastases and optimal strategy for neck dissection. Ann Surg Oncol 2008;15:1177-82.

10. Polistena A, Monacelli M, Lucchini R, et al. Surgical morbidity of cervical lymphadenectomy for thyroid cancer: A retrospective cohort study over 25 years. Int J Surg 2015;21:128-34.

11. Ito Y, Miyauchi A. Lateral and mediastinal lymph node dissection in differentiated thyroid carcinoma: Indications, benefits, and risks. World J Surg 2007;31:905-15.

12. Sturgeon C, Yang A, Elaraj D. Surgical Management of Lymph Node Compartments in Papillary Thyroid Cancer. Surg Oncol Clin N Am 2016;25:17-40.

13. Vayisoglu Y, Ozcan C. Involvement of level IIb lymph node metastasis and dissection in thyroid cancer. Gland Surg 2013;2:180-5.

14. Madenci AL, Caragacianu D, Boeckmann JO, et al. Lateral neck dissection for well-differentiated thyroid carcinoma: A systematic review. Laryngoscope 2014;124:1724-34.

15. Zhang XJ, Liu D, Xu DB, et al. Should level V be included in lateral neck dissection in treating papillary thyroid carcinoma? World J Surg Oncol 2013;25;11:304.

16. Park JY, Koo BS. Individualized optimal surgical extent of the lateral neck in papillary thyroid cancer with lateral cervical metastasis. Eur Arch Otorhinolaryngol
2014;271:1355-60.

17. Russ G, Bonnema SJ, Erdogan MF, et al. European Thyroid Association Guidelines for Ultrasound Malignancy Risk Stratification of Thyroid Nodules in Adults: The EU-TIRADS. Eur Thyroid J 2017;6:225-37.

18. Kim SM, Kim HK, Kim KJ, et al. Thyroidectomy without lateral neck dissection for papillary thyroid carcinoma with lateral neck lymph node metastases and negative intraoperative frozen section. Head Neck 2016;38:285-9.

19. Xu JJ, Yu E, McMullen C, et al. Patterns of regional recurrence in papillary thyroid cancer patients with lateral neck metastases undergoing neck dissection. J Otolaryngol Head Neck Surg 2017;46:43.

20. McNamara WF, Wang LY, Palmer FL, et al. Pattern of neck recurrence after lateral neck dissection for cervical metastases in papillary thyroid cancer. Surgery 2016;159:1565-71.

21. Strajina V, Dy BM, McKenzie TJ, et al. Comprehensive Lateral Neck Dissection in Papillary Thyroid Carcinoma may Reduce Lateral Neck Recurrence Rates. Ann Surg Oncol 2019;26:86-92.

22. Lombardi D, Paderno A, Giordano D, et al. Therapeutic lateral neck dissection in well-differentiated thyroid cancer: Analysis on factors predicting distribution of positive nodes and prognosis. Head Neck 2018;40:242-50.

23. Stack BC, Ferris RL, Goldenberg D, et al. American Thyroid Association Consensus Review and Statement Regarding the Anatomy, Terminology, and Rationale for Lateral Neck Dissection in Differentiated Thyroid Cancer. Thyroid 2012;22:501-8.

24. So YK, Kim MJ, Kim S, et al. Lateral lymph node metastasis in papillary thyroid carcinoma: A systematic review and meta-analysis for prevalence, risk factors, and location. Int J Surg 2018;50:94-103.

25. Périé S, Lesnik M, Samaha S, et al. How to release neck dissections: Role of the triangle between the spinal accessory nerve and the internal jugular vein. Eur Ann Otorhinolaryngol Head Neck Dis 2017;134:201-3.

26. Lee BJ, Wang SG, Lee JC, et al. Level IIb lymph node metastasis in neck dissection for papillary thyroid carcinoma. Arch Otolaryngol Head Neck Surg 2007;133:1028-30.

27. Gong Y, Yang J, Yan S, et al. Pattern of and clinicopathologic risk factors for lateral lymph node metastases in papillary thyroid carcinoma patients with 
lateral cervical lymphadenopathy. Medicine (Baltimore) 2018;97:e12263.

28. Ryu YJ, Cho JS, Yoon JH, et al. Identifying risk factors for recurrence of papillary thyroid cancer in patients who underwent modified radical neck dissection. World J Surg Oncol 2018;16:205.
Cite this article as: Chebib E, Eymerit C, Chabbert-Buffet N, Angelard B, Lacau St Guily J, Périé S. High rate of IIA/ IIB neck groups involvement supports complete lateral neck dissection in thyroid carcinoma. Gland Surg 2020;9(6):1973-1981. doi: 10.21037 /gs-20-443 